\title{
AN ANALYTICAL APPROACH FOR VARIANCE SWAPS WITH AN ORNSTEIN-UHLENBECK PROCESS
}

\author{
JIAN-PENG CAO ${ }^{1}$ and YAN-BING FANG ${ }^{凶 1}$
}

(Received 27 February, 2016; accepted 7 November, 2016; first published online 19 July 2017)

\begin{abstract}
Pricing variance swaps have become a popular subject recently, and most research of this type come under Heston's two-factor model. This paper is an extension of some recent research which used the dimension-reduction technique based on the Heston model. A new closed-form pricing formula focusing on a log-return variance swap is presented here, under the assumption that the underlying asset prices can be described by a meanreverting Gaussian volatility model (Ornstein-Uhlenbeck process). Numerical tests in two respects using the Monte Carlo (MC) simulation are included. Moreover, we discuss a procedure of solving a quadratic differential equation with one variable. Our method can avoid the previously encountered limitations, but requires more time for calculation than other recent analytical discrete models.
\end{abstract}

2010 Mathematics subject classification: 91B70.

Keywords and phrases: variance swaps, Ornstein-Uhlenbeck process, closed-form solution, stochastic volatility.

\section{Introduction}

As the financial market becomes more and more internationalized, the financial problems in some local areas are very likely to spread and become a serious global issue. The closing down of Barings Bank in 1955 serves as a classic example. In addition, the Asian financial crisis in 1997 also caused a series of strong fluctuations in financial circles in Asia, Europe and America. Thus, the financial market represents obvious volatility as the price of stocks rises and drops sharply. Market practitioners ranging from individuals to financial institutes and pension funds, have strong interests in trading future realized volatility or variance against the current implied volatility or variance and hedging risks effectively, because high volatility or variance means high risks and high risks mean high profits. In this financial background, the corresponding volatility derivatives play an important role. The most accepted and popular derivatives are volatility and variance swaps.

\footnotetext{
${ }^{1}$ School of Mathematics and Statistics, Ningxia University, Yinchuan, 750021, China; e-mail: caoxiaojian0524@gmail.com,nxfangzi@163.com.

(C) Australian Mathematical Society 2017, Serial-fee code 1446-1811/2017 \$16.00
} 
Generally, there are two types of volatility and variance swaps products. The historical-variance-based volatility derivatives include products whose payoff depends on the realized variance of the underlying asset. The other type of volatility products is the implied volatility-based products, like the VIX (volatility index) futures traded in the Chicago board options exchange (CBOE). In this paper, we will focus on the former with realized variance being sampled discretely.

Although market practitioners did not begin to trade variance and volatility swaps until the 1990s, considerable research interests have been drawn in terms of developing appropriate valuation approaches and trading strategies. In this paper, we focus on the valuation approaches for variance swaps, which are generally classified into two types: numerical and analytical methods.

There are two subcategories of the analytical methods. In one subcategory, the most influential pioneer works were conducted by Carr and Madan [9] and Demeterfi et al. [10]. Carr and Madan [9] proposed various methods of trading the realized volatility, such as taking a static position in options, delta-hedging an option position, and so on. Demeterfi et al. [10] provided a nice review on the pricing behaviour and theory of both variance and volatility swaps. However, as pointed out by Carr and Corso [6], the drawback of this replication strategy is that it assumes a continuous sampling time of the variance swap, which does not accord with what the actual financial market tells us. In this way, the results of the method can only be regarded as an approximation of the actual cases in financial practice, whose sampling time is discrete. The second kind of analytical methods are the stochastic volatility models. Grunbichler and Longstaff [13] first developed an analytical method for volatility futures based on a mean-reverting squared-root volatility process. Heston [15] derived an analytical solution for both variance and volatility swaps based on the generalized autoregressive conditional heteroskedasticity $(\mathrm{GARCH})$ volatility process. Brockhaus and Long [5] provided an analytical approximation for the pricing of volatility swaps. Other typical examples of these studies include those of Javaheri et al. [16], Swishchuk [27], Carr and Lee [7, 8], Elliott et al. [11] and Sepp [25]. Most of these stochastic volatility models share a limitation: their results are based on the assumption that the realized variance is defined by a continuously sampling approximation, which may fall short of providing pricing results with sufficient accuracy when the actual discrete sampling becomes less frequent.

Numerical methods, as an alternative, have also been developed quite recently. Little and Pant [19] developed a finite-difference approach for the valuation of the discretely sampled variance swaps in an extended Black-Scholes framework with a local volatility function. Windcliff et al. [28] also explored a numerical algorithm to evaluate discretely sampled volatility derivatives using a numerical partial integro-differential equation approach, which can be regarded as an improving the previous pricing algorithm for the discretely sampled volatility derivatives by allowing jumps in the asset price process. Although the two numerical methods based on discretely sampled realized variance achieve high accuracy, their models do not incorporate stochastic volatilities. To remedy this drawback, Little and Pant [19] and 
Windcliff et al. [28] pointed out, respectively, in the conclusions of their papers, that for better pricing and hedging general variance swaps, one needs to adopt an appropriate model that incorporates the stochastic volatility characteristics observed in financial markets.

Very recently, Broadie and Jain [4] presented a closed-form solution for volatility as well as variance swaps with discrete sampling based on integrating the underlying stochastic processes directly, which can be used in a variety of situations such as the Black-Scholes model [3], the Heston stochastic model [14], the Merton jumpdiffusion model [20] and the Bates and Scott stochastic volatility and jump model [2, 24]. Under Heston's two-factor stochastic model, there are a variety of approaches to obtain a closed-form formula for variance swaps based on discretely sampled realized variance. Inspired by Little and Pant [19], Zhu and Lian [31, 32] used the dimensionreduction technique [19] on the basis of a Fourier transform to solve a two-stage stochastic differential equation (SDE) and thus obtaining an analytical solution of Raccati equations. Zhu and Lian [33] also used the expectation of the characteristic function to solve the same question based on the Feynman-Kac formula [12]. In addition, by the joint moment function, Zheng and Kwok [30] obtained a much simpler form of the analytical method to price variance swaps and used it in other applications of generalized variance swaps such as gamma swaps, corridor variance swaps and conditional variance swaps. In addition to the characteristic function, Rujivan and Zhu [22, 23] regarded general assets and their logarithmic form as a contingent claim directly to obtain the analytical solution of Riccati equations. However, when put into the operation of log-return realized variance, their method makes the equations more complex. Lian et al. [18] took advantage of a Taylor series to obtain the approximation form of the characteristic function, and then used the analytical form of variance swaps to get the closed-form formula of volatility swaps, variance options and volatility options.

In this paper, the dimension-reduction technique is used to price variance swaps based on the mean-reverting Gaussian volatility model, that is, the OrnsteinUhlenbeck process which was applied in the Heston model by Little and Pant [19] and Zhu and Lian [32], and was later used by Jia et al. [17] to price actual-return realized variance swaps. This paper can be regarded as an extension of [17, 19, 32], in which the referring method is used to price log-return realized variance. In the meantime, we further clarify that this method can be used in many situations where the distribution of the variance or volatility can be calculated to price discretely sampled variance swaps.

The rest of this paper is organized as follows. In Section 2, the Ornstein-Uhlenbeck process and variance swaps are introduced, followed by the analytical approach for the variance swaps. In Section 3, several numerical tests, as well as the correctness of our solution from various aspects, are demonstrated. Moreover, a discussion of a procedure to solve a quadratic differential equation with one variable is shown, which has been also mentioned in some previous articles [27, 29]. Finally, Section 4 concludes the paper. 


\section{Our model}

In this section, we briefly review the mean-reverting Gaussian volatility model to describe the dynamics of the underlying asset. Then, we use the Fourier transform to obtain the associated partial differential equation (PDE), and arrive at the analytical solution, based on the dimension-reduction technique proposed by Little and Pant [19].

2.1. The mean-reverting Gaussian volatility model Considering that volatility follows an Ornstein-Uhlenbeck process, as assumed by Stein and Stein [26], the stochastic process of the underlying asset price $S_{t}$ and the stochastic instantaneous volatility $v_{t}$ can be described as

$$
\left\{\begin{array}{l}
d S_{t}=\mu S_{t} d t+v_{t} S_{t} d B_{t}^{S} \\
d v_{t}=\kappa\left(\theta-v_{t}\right) d t+\sigma d B_{t}^{v}
\end{array}\right.
$$

where $\mu$ is the expected return of the underlying asset, $\theta$ is the long-term mean of volatility, $\kappa$ is the mean-reverting speed parameter of the volatility, $\sigma$ is the so-called volatility of volatility. The two Wiener processes $d B_{t}^{S}$ and $d B_{t}^{v}$ describe the random noise in asset and volatility, respectively. They are assumed to be correlated with a constant correlation coefficient $\rho$, that is, $\left(d B_{t}^{S}, d B_{t}^{v}\right)=\rho d t$.

Under no arbitrage there exists a risk-neutral measure $\mathbb{Q}$, such that the prices of all nondividend paying assets are martingales under this measure. In this measure, we can describe the process as

$$
\left\{\begin{array}{l}
d S_{t}=r S_{t} d t+v_{t} S_{t} d \widetilde{B}_{t}^{S}, \\
d v_{t}=\kappa^{*}\left(\theta^{*}-v_{t}\right) d t+\sigma d \widetilde{B}_{t}^{v},
\end{array}\right.
$$

where $r$ denotes the riskless interest rate, $\kappa^{*}=\kappa+\lambda$ and $\theta^{*}=\kappa \theta / \kappa+\lambda$ are the riskneutral parameters, the new parameter $\lambda$ is the premium of volatility risk. In the rest of the paper, we focus on a risk-neutral probability measure.

2.2. Variance swaps A variance swap is a derivative contract that pays at a fixed maturity $T$, the difference between a given level (fixed leg) and a realized level of variance over the swaps life (floating leg). They can be useful for hedging volatility risk exposure or for taking positions on future realized volatility. When a maturity is given, such as $T>0$, the payoff of a variance swap can be written as $V_{T}=\left(\sigma_{R}^{2}-K_{\mathrm{var}}\right) \cdot L$, where $\sigma_{R}^{2}$ is the annualized realized variance over the contract time $[0, T], K_{\mathrm{var}}$ is the annualized delivery price for the variance swap, and $L$ is the notional amount of the swap in dollars per annualized volatility point squared.

Classically, there are two methods to calculate discretely-sampled variance swaps (see [30-33]), which can be categorized into two different definitions: the log-return realized variance defined by

$$
\sigma_{R, d 1}^{2}(0, N, T)=\frac{A F}{N} \sum_{i=1}^{N} \log ^{2}\left(\frac{S_{t_{i}}}{S_{t_{i-1}}}\right) 100^{2}=\frac{1}{T} \sum_{i=1}^{N} \log ^{2}\left(\frac{S_{t_{i}}}{S_{t_{i-1}}}\right) 100^{2},
$$


and the actual return-based realized variance defined by

$$
\sigma_{R, d 2}^{2}(0, N, T)=\frac{A F}{N} \sum_{i=1}^{N}\left(\frac{S_{t_{i}}-S_{t_{i-1}}}{S_{t_{i-1}}}\right)^{2} 100^{2}=\frac{1}{T} \sum_{i=1}^{N}\left(\frac{S_{t_{i}}-S_{t_{i-1}}}{S_{t_{i-1}}}\right)^{2} 100^{2},
$$

where $S_{t_{i}}$ is the closing price of the underlying asset at the $i$ th observation time $t_{i}$, and there are $N$ observations in all. $A F$ is the annualized factor converting this expression to an annualized variance. If the sampling frequency is every trading day then $A F=252$, (here we assume that there are 252 trading days in one year), if every week then $A F=52$, if every month then $A F=12$, and so on. Typically, we set $T=N / A F$ and assume equally-spaced discrete observations, so that the annualized factor is of a simple expression $A F=N / T=1 / \Delta t$.

In the risk-neutral world, the payoff of a variance swap at time $t$ is the expected present value of the future payoff $V_{t}=\mathbb{E}_{t}^{Q}\left[\exp ^{-r(T-t)}\left(\sigma_{R}^{2}-K_{\mathrm{var}}\right) L\right]$, where $Q$ is the riskneural probability measure, $\mathbb{E}_{t}^{Q}[\cdot]=\mathbb{E}^{Q}\left[\cdot \mid \mathcal{A}_{t}\right]$ is the conditional expectation at time $t$ and $\mathcal{A}_{t}$ is the filtration up to time $t$. The price of variance swaps should be zero at the beginning of the contract, since there is no cost for either party to enter into a swap contract. Therefore, the fair variance delivery price is easily defined as $K=\mathbb{E}_{0}^{Q}\left[\sigma_{R}^{2}\right]$. The valuation problem for a variance swap is, therefore, reduced to calculating the expectation value of the future realized variance in the risk-neutral world. Jia et al. [17] solved the analytical solution of the expectation with respect to (2.3), using the method of Little and Pant [19]. In this paper, we will focus on the expectation of log-return realized variance in (2.2).

2.3. Pricing approach for variance swaps As mentioned above, the solution of our approach to price variance swaps can be regarded as finding out the expectation of $\sigma_{R, d 1}^{2}$, which can be defined as

$$
\mathbb{E}_{0}^{Q}\left[\sigma_{R, d 1}^{2}(0, N, T)\right]=\mathbb{E}_{0}^{Q}\left[\frac{A F}{N} \sum_{i=1}^{N} \log ^{2}\left(\frac{S_{t_{i}}}{S_{t_{i-1}}}\right)\right] 100^{2}=\frac{100^{2}}{N \Delta t} \sum_{i=1}^{N} \mathbb{E}_{0}^{Q}\left[\log ^{2}\left(\frac{S_{t_{i}}}{S_{t_{i-1}}}\right)\right] .
$$

Thus, this problem can be reduced to calculating $N$ expectations in the form

$$
\mathbb{E}_{0}^{Q}\left[\log ^{2}\left(\frac{S_{t_{i}}}{S_{t_{i-1}}}\right)\right]
$$

for some fixed time period $\Delta t$ and $N$ different tenors $t_{i}=i \Delta t(i=1, \ldots, N)$. In the next part, the expectation in equation (2.5) will be focused on. Consider $i$ as a constant; thus, tenors $t_{i}$ and $t_{i-1}$ are also constants. We assume that the current time is $t_{0}$.

There are two kinds of situations: $i>1$ and $i=1$. Because $S_{t_{i-1}}$ is known when $i=1$, we just need to take the expectation of one variable, while in the former there are two variables, which makes it difficult to solve the expectations of them simultaneously. We start with the former situation. A new variable defined as a Dirac delta function $I_{t}$ is introduced as

$$
I_{t}=\int_{0}^{t} \delta\left(t_{i-1}-\tau\right) S_{\tau} d \tau= \begin{cases}S_{t_{i-1},} & t_{i-1} \leq t \leq t_{i} \\ 0, & 0 \leq t<t_{i-1}\end{cases}
$$


The property of the Dirac delta function will be used to turn the two-variable expectation into two one-variable expectations in the next part of this section. Considering a contingent claim $U_{i}=U_{i}(S, v, I, t)$ whose payoff at expiry $t_{i}$ is $\log ^{2}\left(S_{t_{i}} / I_{t_{i}}\right)$, a PDE for $U_{i}$ can be obtained according to general asset valuation theory [12], that is,

$$
\begin{aligned}
& \frac{\partial U_{i}}{\partial t}+\frac{1}{2} v^{2} S^{2} \frac{\partial^{2} U_{i}}{\partial S^{2}}+\rho \sigma v S \frac{\partial^{2} U_{i}}{\partial S \partial v} \\
& +\frac{1}{2} \sigma^{2} \frac{\partial^{2} U_{i}}{\partial v^{2}}+r S \frac{\partial U_{i}}{\partial S}+\left[\kappa^{*}\left(\theta^{*}-v\right)\right] \frac{\partial U_{i}}{\partial v}-r U_{i}+\delta\left(t_{i-1}-t\right) \frac{\partial U_{i}}{\partial I}=0
\end{aligned}
$$

with terminal condition

$$
U_{i}\left(S_{t_{i}}, v_{t_{i}}, I_{t_{i}}, t_{i}\right)=\log ^{2}\left(\frac{S_{t_{i}}}{I_{t_{i}}}\right) .
$$

From the Feynman-Kac theorem [12], it is known that $U_{i}\left(S_{t_{i}}, v_{t_{i}}, I_{t_{i}}, t_{i}\right)$ has the property

$$
\mathbb{E}_{0}^{Q}\left[U_{i}\left(S_{t_{i}}, v_{t_{i}}, I_{t_{i}}, t_{i}\right)\right]=e^{r t_{i}} U_{i}\left(S_{0}, v_{0}, I_{0}, 0\right) .
$$

This formula establishes a relationship between certain variables such as $v_{0}$ at time 0 and $v_{t_{i}}$ at time $t_{i}$. We will begin at time $t_{i}$, and obtain the analytical solution at time $t_{i-1}$, then at time 0. Finally, the conditional expectation in (2.5) is solved by using (2.8). Since at time $t \neq t_{i-1}, \delta\left(t_{i-1}-t\right)=0$, equation (2.6) can be rewritten as:

$$
\frac{\partial U_{i}}{\partial t}+\frac{1}{2} v^{2} S^{2} \frac{\partial^{2} U_{i}}{\partial S^{2}}+\rho \sigma v S \frac{\partial^{2} U_{i}}{\partial S \partial v}+\frac{1}{2} \sigma^{2} \frac{\partial^{2} U_{i}}{\partial v^{2}}+r S \frac{\partial U_{i}}{\partial S}+\left[\kappa^{*}\left(\theta^{*}-v\right)\right] \frac{\partial U_{i}}{\partial v}-r U_{i}=0
$$

Although the simplified PDE ignores the influence of variable $I$, it is not ignored in equation (2.7). In the meantime, because of the property of $I$, it should be considered in two situations: $t<t_{i-1}$ and $t \geq t_{i-1}$. From the property of $I$ it is known that the value of $I$ is a jump as time increases from 0 to $t_{i-1}$, its value turns from 0 into $S_{t_{i-1}}$. Because of the no-arbitrary assumption, the value of $U_{i}$ cannot jump at time $t_{i-1}$. Mathematically, the jump condition can be written as:

$$
\lim _{t \uparrow t_{i-1}} U_{i}(S, v, I, t)=\lim _{t \downarrow t_{i-1}} U_{i}(S, V, I, t) .
$$

The original expectation has two unknown variables; theoretically, one of them can be solved in one step only. We may as well use the property of $I$ and $U$ to divide the above PDE into two stages. The time can be divided into two ranges $\left[0, t_{i-1}\right]$ and $\left[t_{i-1}, t_{i}\right]$, in each of which $I$ can be regarded as a constant, because its value does not change. Thus, the PDE can be solved in $\left[t_{i-1}, t_{i}\right]$ first, and then in $\left[0, t_{i}\right]$. Accordingly, the PDE can be expressed by two systems as follows:

$$
\left\{\begin{array}{c}
\frac{\partial U_{i}}{\partial t}+\frac{1}{2} v^{2} S^{2} \frac{\partial^{2} U_{i}}{\partial S^{2}}+\rho \sigma v S \frac{\partial^{2} U_{i}}{\partial S \partial v}+\frac{1}{2} \sigma^{2} \frac{\partial^{2} U_{i}}{\partial v^{2}} \\
+r S \frac{\partial U_{i}}{\partial S}+\left[\kappa^{*}\left(\theta^{*}-v\right)\right] \frac{\partial U_{i}}{\partial v}-r U_{i}=0 \\
U_{i}\left(S, v, I, t_{i}\right)=\log ^{2}\left(\frac{S}{I}\right), \quad t_{i-1} \leq t \leq t_{i}
\end{array}\right.
$$


and

$$
\left\{\begin{array}{l}
\frac{\partial U_{i}}{\partial t}+\frac{1}{2} v^{2} S^{2} \frac{\partial^{2} U_{i}}{\partial S^{2}}+\rho \sigma v S \frac{\partial^{2} U_{i}}{\partial S \partial v}+\frac{1}{2} \sigma^{2} \frac{\partial^{2} U_{i}}{\partial v^{2}} \\
\quad+r S \frac{\partial U_{i}}{\partial S}+\left[\kappa^{*}\left(\theta^{*}-v\right)\right] \frac{\partial U_{i}}{\partial v}-r U_{i}=0, \\
\lim _{t \uparrow t_{i-1}} U_{i}(S, v, I, t)=\lim _{t \downarrow t_{i-1}} U_{i}(S, V, I, t), \quad 0 \leq t \leq t_{i-1} .
\end{array}\right.
$$

Note that system (2.9) should be solved first, and then the limit condition in (2.10) may be used to solve the next system. The generalized Fourier transform method (see [21]) will be utilized to solve the first system.

Proposition 2.1. If the underlying asset follows the dynamic process (2.1) and a European-style derivative written on this underlying asset has a payoff function $U(S, v, T)=H(S)$ at expiry $T$, then the solution of the corresponding PDE system of the derivative

$\left\{\begin{array}{l}\frac{\partial U}{\partial t}+\frac{1}{2} v^{2} S^{2} \frac{\partial^{2} U}{\partial S^{2}}+\rho \sigma v S \frac{\partial^{2} U}{\partial S \partial v}+\frac{1}{2} \sigma^{2} \frac{\partial^{2} U}{\partial v^{2}}+r S \frac{\partial U}{\partial S}+\left[\kappa^{*}\left(\theta^{*}-v\right)\right] \frac{\partial U}{\partial v}-r U=0, \\ U(S, v, T)=H(S)\end{array}\right.$

can be expressed as

$$
U(x, v, t)=\mathcal{F}^{-1}\left[e^{C(\omega, T-t)+D(\omega, T-t) v+E(\omega, T-t) v^{2}} \mathcal{F}\left[H\left(e^{x}\right)\right]\right],
$$

where

$$
\left\{\begin{aligned}
E(\omega, \tau)= & \frac{c_{1}\left(-1+e^{2 B \tau}\right)}{A\left(-1+e^{2 B \tau}\right)+B\left(1+e^{2 B \tau}\right)}, \\
D(\omega, \tau)= & -\frac{2 c_{1}\left(-1+e^{B \tau}\right)^{2} \theta^{*} \kappa^{*}}{B\left\{A\left(-1+e^{2 B \tau}\right)+B\left(1+e^{2 B \tau}\right)\right\}}, \\
C(\omega, \tau)= & \frac{1}{2} \log \frac{A\left(-1+e^{2 B \tau}\right)+B\left(1+e^{2 B \tau}\right)}{2 B}+\tau\left(b-\frac{B+A}{2}-\frac{c_{1} \theta^{* 2} \kappa^{* 2}}{B^{2}}\right) \\
& +\frac{\theta^{* 2} \kappa^{* 2} c_{1}\left\{2 A\left(-1+e^{B \tau}\right)^{2}+B\left(-1+e^{2 B \tau}\right)\right\}}{B^{3}\left\{A\left(-1+e^{2 B \tau}\right)+B\left(1+e^{2 B \tau}\right)\right\}},
\end{aligned}\right.
$$

and $A=\kappa^{*}-i \rho \sigma \omega, c_{1}=\left(\omega^{2}+i \omega\right) / 2, \quad B=\sqrt{A^{2}+2 c_{1} \sigma^{2}}, \quad b=(i \omega-1) r, \quad i=\sqrt{-1}$, $\tau=T-t, x=\ln S, \omega$ is the Fourier transform variable.

The proof of this proposition is given in Appendix A.

We believe that there might be other forms of the payoff function. However, whether there are other suitable forms of the payoff function or not will be put aside now. All of the previous research has paid attention to only one form (A2). Although recently certain papers $[22,29]$ pointed out a limitation to this form, in this paper, we examine the correctness of (A2) in the payoff function. At the end of this section, we discuss the payoff function in our model as well as [29]. 
Before getting the solution in Proposition 2.1 based on the inverse Fourier transform, we first introduce a characteristic of the Fourier transform and Fourier inverse transform.

Lemma 2.2. Based on the generalized Fourier transform, we can perform the transformation as

$$
\mathcal{F}\left[x^{n}\right]=2 \pi i^{n} \delta^{(n)}(\omega),
$$

where $\delta^{(n)}$ is the nth order derivative of the generalized delta function satisfying

$$
\int_{-\infty}^{\infty} \delta^{(n)}(\omega) \Phi(\omega) d \omega=(-1)^{n} \Phi^{(n)}(0) .
$$

Note that $I$ is a constant, and $H(S)=\log ^{2}(S / I)$. The generalized Fourier transform of $H(x)$ with respect to $x$ is given by

$$
\mathcal{F}\left[(x-\log I)^{2}\right]=2 \pi\left[-\delta^{(2)}(\omega)-2 i \delta^{(1)}(\omega) \log I+\delta(\omega) \log ^{2} I\right] .
$$

Using Lemma 2.2, the solution of the PDE (2.9) is obtained as

$$
\begin{aligned}
U_{i}(S, v, I, t)= & \mathcal{F}^{-1}\left[e ^ { C ( \omega , t _ { i } - t ) + D ( \omega , t _ { i } - t ) v + E ( \omega , t _ { i } - t ) v ^ { 2 } } 2 \pi \left[-\delta^{(2)}(\omega)\right.\right. \\
& \left.\left.-2 i \delta^{(1)}(\omega) \log I+\delta(\omega) \log ^{2} I\right]\right] \\
= & \int_{-\infty}^{\infty}\left[e ^ { C ( \omega , t _ { i } - t ) + D ( \omega , t _ { i } - t ) v + E ( \omega , t _ { i } - t ) v ^ { 2 } } \left\{-\delta^{(2)}(\omega)\right.\right. \\
& \left.\left.-2 i \delta^{(1)}(\omega) \log I+\delta(\omega) \log ^{2} I\right\} e^{x \omega i}\right] d \omega \\
= & -f^{(2)}(0)+2 i f^{(1)}(0) \log I+f(0) \log ^{2} I,
\end{aligned}
$$

where

$$
f(\omega)=e^{C\left(\omega, t_{i}-t\right)+D\left(\omega, t_{i}-t\right) v+E\left(\omega, t_{i}-t\right) v^{2}+x \omega i}, \quad f^{(1)}(0)=\left.\frac{d f(\omega)}{d \omega}\right|_{\omega=0}, \quad f^{(2)}(0)=\left.\frac{d^{2} f(\omega)}{d \omega^{2}}\right|_{\omega=0} .
$$

Up to now, the first stage to solve system (2.9) has been done by the Fourier transform and the inverse Fourier transform. To finish off the calculation of $\mathbb{E}_{0}^{Q}\left[\log ^{2}\left(S_{t_{i}} / S_{t_{i-1}}\right)\right]$, the system (2.10) needs to be solved.

Using the condition $\lim _{t \downarrow t_{i-1}} \log S_{t}=\log I$, which is derived from the definition of $I$, we take the limit on the left hand side of equation (2.12) to obtain

$$
\begin{aligned}
\lim _{t \downarrow t_{i-1}} U_{i}(S, v, I, t)= & e^{-r \Delta t} g(v) \\
= & e^{-r \Delta t}\left[-\left(C^{(1)}(0)\right)^{2}-2 v C^{(1)}(0) D^{(1)}(0)-v^{2}\left(D^{(1)}(0)\right)^{2}\right. \\
& -2 v^{2} C^{(1)}(0) E^{(1)}(0)-2 v^{3} D^{(1)}(0) E^{(1)}(0)-v^{4}\left(E^{(1)}(0)\right)^{2} \\
& \left.-C^{(2)}(0)-v D^{(2)}(0)-v^{2} E^{(2)}(0)\right]
\end{aligned}
$$

where

$$
C^{(1)}(0)=\left.\frac{\partial C(\omega, \Delta t)}{\partial \omega}\right|_{\omega=0}, \quad C^{(2)}(0)=\left.\frac{\partial^{2} C(\omega, \Delta t)}{\partial \omega^{2}}\right|_{\omega=0} ;
$$

$D$ and $E$ are defined similarly; $C(\omega, \tau), D(\omega, \tau)$ and $E(\omega, \tau)$ have been given in (2.11). 
At this stage, it may seem that the value of $U_{i}(S, v, I, t)$ will become complex, since all parameters in (2.1) are real and it is obvious that all the derivatives of $A, B, c_{1}, b$ with respect to $\omega=0$ are complex, such as

$$
\left.\frac{\partial B}{\partial \omega}\right|_{\omega=0}=\frac{i\left(\sigma^{2}-2 \sigma \kappa \rho\right)}{2 \kappa} \neq 0 .
$$

Due to the complexity of analytic formulas $C^{(1)}(0), D^{(1)}(0), E^{(1)}(0), C^{(2)}(0)$ and so on, it is necessary to directly give these formulas, but we indicate that the value of $U_{i}(S, v, I, t)$ is not complex from another point of view. Before using the Fourier transform and the Fourier inverse transform to obtain the analytical formula of $U_{i}(S, v, I, t)$, we assume that the analytical form of $U_{i}(S, v, I, t)$ exists in (2.9), which contains no term with $i$, because all pricing models are built on a real number field according to their practical meaning. It is absolutely true that $\mathcal{F}^{-1}\left[\mathcal{F}\left[U_{i}(S, v, I, t)\right]\right]=$ $U_{i}(S, v, I, t)$. Thus, (2.13) is actually built on a real number field, and the last number of it is real even if some terms involve the coefficient $i$. Put another way, the derivative (2.13) exists in real number field when $\omega=0$ (see Zhu and Lian [32]).

The initial value condition in (2.10) is a function with independent value $v$. Later, the distribution of $v$ will be obtained and will be used to solve system (2.11).

Proposition 2.3. If the underlying asset follows the dynamic (2.1) and a Europeanstyle derivative written on this underlying asset has a payoff function $U(S, v, T)=$ $G\left(v_{T}\right)$ at expiry $T$, then the solution of the corresponding PDE system of the derivative

$$
\left\{\begin{array}{c}
\frac{\partial U}{\partial t}+\frac{1}{2} v^{2} S^{2} \frac{\partial^{2} U}{\partial S^{2}}+\rho \sigma v S \frac{\partial^{2} U}{\partial S \partial v}+\frac{1}{2} \sigma^{2} \frac{\partial^{2} U}{\partial v^{2}} \\
+r S \frac{\partial U}{\partial S}+\left[\kappa^{*}\left(\theta^{*}-v\right)\right] \frac{\partial U}{\partial v}-r U=0 \\
U(S, v, T)=G(v)
\end{array}\right.
$$

can be denoted in the form

$$
U(S, v, t)=\int_{-\infty}^{\infty} e^{-r(T-t)} G\left(v_{T}\right) p\left(v_{T} \mid v_{t}\right) d v_{T},
$$

where

$$
\left\{\begin{array}{l}
p\left(v_{t} \mid v_{0}\right)=\frac{1}{\sqrt{2 \pi} \hat{\sigma}(t)} e^{-\left(v_{t}-\mu(t)\right)^{2} / 2 \hat{\sigma}(t)^{2}} \\
\mu(t)=e^{-\kappa^{*} t} v_{0}+\theta^{*}\left(1-e^{-\kappa^{*} t}\right) \\
\hat{\sigma}(t)^{2}=\frac{\sigma^{2}}{2 \kappa^{*}}\left(1-e^{-2 \kappa^{*} t}\right) .
\end{array}\right.
$$

The proof of this proposition is in Appendix B.

According to equations (2.15) and (2.13), for $0 \leq t<t_{i-1}$,

$$
U_{i}(S, v, I, t)=\int_{-\infty}^{\infty} e^{-r\left(t_{i-1}-t\right)-r \Delta t} g\left(v_{t_{i-1}}\right) p\left(v_{t_{i-1}} \mid v_{t}\right) d v_{t_{i-1}} .
$$


Then we use (2.7) and (2.8) based on the Feynman-Kac theorem to arrive at the calculation of expectation (2.5) as

$$
\mathbb{E}_{0}^{Q}\left[\log ^{2}\left(\frac{S_{t_{i}}}{S_{t_{i-1}}}\right)\right]=e^{r t_{i}} U_{i}\left(S_{0}, v_{0}, I_{0}, 0\right)=\int_{-\infty}^{\infty} g\left(v_{t_{i-1}}\right) p\left(v_{t_{i-1}} \mid v_{0}\right) d v_{t_{i}} .
$$

Besides variable $v_{t}$, we need to get the conditional expectation of $v_{t}^{2}, v_{t}^{3}$ and $v_{t}^{4}$ in $g\left(v_{t}\right)$. Thus, the expectations of the last three variables should be solved. Using $\mathbb{D}\left(v_{t}\right)=\mathbb{E}\left(v_{t}^{2}\right)-\left[\mathbb{E}\left(v_{t}\right)\right]^{2}$, the expectation

$$
\mathbb{E}_{0}^{Q}\left(v_{t}^{2}\right)=\mu(t)^{2}+\hat{\sigma}(t)^{2} .
$$

Let $k_{t}=\left(v_{t}-\mu(t)\right) / \hat{\sigma}(t)$, which has a standard normal distribution. Since $E_{0}^{Q}\left(k_{t}^{3}\right)=0$,

$$
\mathbb{E}_{0}^{Q}\left(v_{t}^{3}\right)-3 \mathbb{E}_{0}^{Q}\left(v_{t}^{2}\right) \mu(t)+3 \mathbb{E}_{0}^{Q}\left(v_{t}\right) \mu(t)^{2}-\mu(t)^{3}=0 .
$$

Accordingly, the expectation of $v_{t}^{3}$

$$
\mathbb{E}_{0}^{Q}\left(v_{t}^{3}\right)=3 \mu(t) \hat{\sigma}(t)^{2}+\mu(t)^{3} .
$$

In the meantime, note that $k_{t}^{2}$ is chi-squared distributed with mean 1 and variance 2 . Thus, the expectation of $k_{t}^{4}$ is 3 according to (2.16). Then it follows that

$$
\mathbb{E}_{0}^{Q}\left(v_{t}^{4}\right)-4 \mathbb{E}_{0}^{Q}\left(v_{t}^{3}\right) \mu(t)+6 \mathbb{E}_{0}^{Q}\left(v_{t}^{2}\right) \mu(t)^{2}-4 \mathbb{E}_{0}^{Q}\left(v_{t}\right) \mu(t)^{3}+\mu(t)^{4}=3 \hat{\sigma}(t)^{4} .
$$

Based on the results of (2.16) and (2.17), the expectation of $v_{t}^{4}$

$$
\mathbb{E}_{0}^{Q}\left(v_{t}^{4}\right)=3 \hat{\sigma}(t)^{4}+6 \hat{\sigma}(t)^{2} \mu(t)^{2}+\mu(t)^{4} .
$$

After getting the corresponding expectations of certain variables including $v_{t}$, the expectation in (2.5) is obtained as

$$
\mathbb{E}_{0}^{Q}\left[\log ^{2}\left(\frac{S_{t_{i}}}{S_{t_{i-1}}}\right)\right]=g_{i}\left(v_{0}\right),
$$

where

$$
\begin{aligned}
g_{i}\left(v_{0}\right)= & \int_{-\infty}^{\infty} g\left(v_{t_{i-1}}\right) p\left(v_{t_{i-1}} \mid v_{0}\right) d v_{t_{i-1}} \\
= & \left\{-2 C^{(1)}(0) D^{(1)}(0)-D^{(2)}(0)\right\} \mu\left(t_{i-1}\right)+\left\{-\left(D^{(1)}(0)\right)^{2}-2 C^{(1)}(0) E^{(1)}(0)-E^{(2)}(0)\right\} \\
& \times\left\{\mu\left(t_{i-1}\right)^{2}+\hat{\sigma}\left(t_{i-1}\right)^{2}\right\}-2 D^{(1)}(0) E^{(1)}(0)\left\{3 \mu\left(t_{i-1}\right) \hat{\sigma}\left(t_{i-1}\right)^{2}+\mu\left(t_{i-1}\right)^{3}\right\} \\
& -E^{(1)}\left\{3 \hat{\sigma}\left(t_{i-1}\right)^{4}+6 \hat{\sigma}\left(t_{i-1}\right)^{2} \mu\left(t_{i-1}\right)^{2}+\mu\left(t_{i-1}\right)^{4}\right\}-\left(C^{(1)}(0)\right)^{2}-C^{(2)}(0) . \quad(2.20)
\end{aligned}
$$

Using (2.18), the summation in (2.4) can now be carried out, except for the very first period when $i=1$. Finally, the case where $i=1$ needs to be coped with, because in this case $t_{i-1}=0$ and $S_{t_{i-1}}=S_{0}$, which is the current underlying asset price and a known value. Thus, in this case, we just use Proposition 2.1 to get the expectation

$$
\mathbb{E}_{0}^{Q}\left[\log ^{2}\left(\frac{S_{t_{i}}}{S_{t_{i-1}}}\right)\right]=g\left(v_{0}\right)
$$


Based on the solutions of (2.19) and (2.21), the fair strike price for variance swaps

$$
K_{\mathrm{var}}=\mathbb{E}_{0}^{Q}\left[\sigma_{R, d 1}^{2}(0, N, T)\right]=\frac{1}{T}\left[g\left(v_{0}\right)+\sum_{i=2}^{N} g_{i}\left(v_{0}\right)\right] 100^{2},
$$

where $N$ denotes the total sampling times of the swap contract. The above formula is obtained by solving the two associated PDEs, and each one of them focuses on a variable. This method avoids calculating the expectation of the payoff function directly, where the connection between $S_{t_{i}}$ and $S_{t_{i-1}}$ should be found due to the form of the payoff function. When using this dimension reduction technique for price variance swaps under different dynamic processes, all of the first stages are similar and a group of Riccati differential equations [32] should be obtained and solved. In the second phase, the transition probability function of $v_{t}$ at different times should be founded, which is simpler than that of $S_{t}$. Whether this method can take effect in other dynamic processes or not will be discussed in our future research. With the newly found analytical solution, all the hedging ratios of a variance swap are also analytically obtained by taking partial derivatives against various parameters in the model.

At the end of this section, we will discuss some differences among the analytical pricing approaches in several recent articles. The same principles appeared in the work of Zhu and Lian [31], Little and plant [19], Jia et al. [17] and our model. The first difference between them is that the models of the first two articles are based on the Heston model, which means that the volatility $v_{t}$ follows a noncentral chi-square distribution, while $v_{t}$ in the last two models is normally distributed. The advantage of the Heston model in pricing variance swaps is obvious. It can make the derivation process easier than the Ornstein-Uhlenbeck $(\mathrm{O}-\mathrm{U})$ process when solving a group of ordinary differential equations (ODEs). As for the article based on the O-U process such as Zhang [29], which has the same principle as that of Rujivan and Zhu [22, 23], its model has a seemingly easier principle than ours. Except for the distribution of $v_{t}$ and the Feynman-Kac formula [12], our model is much more complex when solving this formula, because the Fourier transform is used to solve it indirectly. In addition, although both of the two models use the Feynman-Kac formula, there is a difference in this formula between the two models. By the Feynman-Kac theorem, the derivative of a contingent claim about the riskless interest rate is included in our model, but not in the other one. But, this indirect method has its advantage and we will talk about it in the following section. Although papers [22, 23, 29] are based on the same principle, which is to regard the determined form of underlying assets as the contingent claim and to put it into the Feynman-Kac formula, there are a few differences between them due to their different applications. When it comes to log-return variance swaps [23], we need to solve a group of ODEs, which is more complex than actual-return variance swaps. However, it will stay in first order instead of second, which means the result of the ODEs has a simpler form than [22, 29]. In addition, its result will not be local. The principle of all of the recent methods to solve the question on the analytical pricing approach with respect to variance swaps, is to avoid solving the expectation of $S_{t_{i}}$ and 
$S_{t_{i-1}}$ directly and to find their transition density function. Instead, they first use the characteristic of the connected dynamic processes of $v_{t}$ and $S_{t}$ to make $S_{t_{i}}$ and $S_{t_{i-1}}$ disappear and then use the distribution of $v_{t}$ to solve this question. In the following section, we also talk about the running time of two different analytical methods.

\section{Numerical tests and discussions}

We now implement our method and present numerical examples to show the accuracy of it, based on the $\mathrm{O}-\mathrm{U}$ stochastic volatility model. There are seven parameters that need to be assigned a value. Since our purpose is to find and verify an exact solution for the valuation and analysis of variance swaps, how the values of these parameters are determined (see [1]) has nothing to do with us. In this section, we use the following values for the parameters: $v_{0}=0.04, \theta^{*}=0.022, \kappa^{*}=11.35$, $\rho=-0.64, \sigma=0.618, r=0.1$ and $T=1$. The method used to test our model is the Monte Carlo (MC) simulation, assuming $S_{0}=1$.

3.1. MC simulation Our aim is to ge at benchmark value for our solution (2.22). In the MC simulation, we employ a simple Euler-Maruyama discretization for the $\mathrm{O}-\mathrm{U}$ process

$$
\left\{\begin{array}{l}
S_{t}=S_{t-1}+r S_{t-1} \Delta t+v_{t-1} S_{t-1} \sqrt{\Delta t} W_{t}^{1}, \\
v_{t}=v_{t-1}+\kappa^{*}\left(\theta^{*}-v_{t-1}\right) \Delta t+\sigma \sqrt{\Delta t}\left(\rho W_{t}^{1}+\sqrt{1-\rho^{2}} W_{t}^{2}\right) .
\end{array}\right.
$$

From Zhang's work [29], we know that the analytical solution of the continuous model with an $\mathrm{O}-\mathrm{U}$ process yields

$$
\begin{aligned}
K_{\infty} & =100^{2} \mathbb{E}_{0}^{Q}\left[\sigma_{R}^{2}\right] d t=\frac{100^{2}}{T} \int_{0}^{T} \mathbb{E}_{0}^{Q}\left[v_{t}^{2}\right] d t=\frac{100^{2}}{T} \int_{0}^{T}\left(\hat{\sigma}(t)^{2}+\mu(t)^{2}\right) d t \\
& =\left[\theta^{* 2}+\frac{\sigma^{2}}{2 \kappa^{*}}+\frac{2 \theta^{*}\left(v_{0}-\theta^{*}\right)}{\kappa^{*} T}\left(1-e^{-k^{*} T}\right)+\frac{\left(v_{0}-\theta^{*}\right)^{2}-\sigma^{2} /\left(2 \kappa^{*}\right)}{2 \kappa^{*} T}\left(1-e^{-2 \kappa^{*} T}\right)\right] 100^{2} .
\end{aligned}
$$

Theoretically, if our model is correct, the values of both the MC simulations and our discrete model should approach the continuous model as sampling frequency increases. (The number of paths reaches 200000 in the MC simulation.) Assuming $T=1$, we verify the correctness of our model by Matlab and present the result in Figure 1. From the graph, it is clear that the value of our model matches the MC simulation very well. Moreover, the two values approach that of continuous approximation when the sampling frequency tends to be infinite. In this paper, we cannot prove that the analytical formula of the continuous model is the limit of the discrete model due to the complexity of our model, which contains derivation process. Even so, our method is at least numerically reasonable by the graph.

To further show the numerical test and the gap between the solution of our model and the benchmark value, we present specific data of the discrete model, the continuous model and the MC simulation in Table 1.

Due to the similarity between the mean-reverting Gaussian volatility model and the Heston model, we can test our analytical solution from another point of view: 


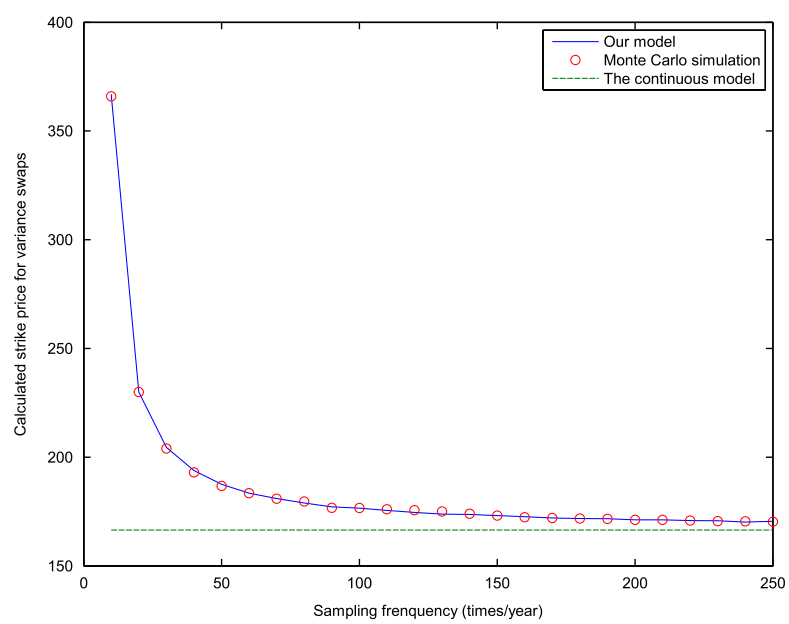

FIGURE 1. A comparison of fair strike values based on the discrete model, continuous model and (3.1).

TABLE 1. The numerical results.

\begin{tabular}{lccc}
\hline Sampling frequency & Discrete model & Continuous model & MC simulation \\
\hline Monthly $(N=12)$ & 309.4316 & 166.5172 & 307.1123 \\
Fortnightly $(N=26)$ & 211.7691 & 166.5172 & 212.5393 \\
Weekly $(N=52)$ & 188.6041 & 166.5172 & 187.4576 \\
Daily $(N=252)$ & 170.5176 & 166.5172 & 170.6220 \\
\hline
\end{tabular}

the Heston model [15]. First of all, we introduce the relationship between the Heston model and the mean-reverting volatility Gaussian model. In the Heston model, the process of the variance of underlying assets is

$$
d \hat{v}_{t}=\hat{\kappa}^{*}\left(\hat{\theta}^{*}-\hat{v}_{t}\right) d t+\hat{\sigma} \sqrt{\hat{v}_{t}} d \widetilde{B}_{t}^{\hat{v}} v .
$$

Since $v_{t}$ in the $\mathrm{O}-\mathrm{U}$ process denotes volatility, $\hat{v}_{t}=v_{t}^{2}$. Using the It $\hat{o}$ formula for $v_{t}^{2}$, the process of $v_{t}^{2}$ is obtained, and substituting $\hat{v}_{t}$ for $v_{t}^{2}$ yields

$$
d \hat{v}_{t}=\left(\sigma^{2}+2 \kappa^{*} \theta^{*} \sqrt{\hat{v}_{t}}-2 \kappa^{*} \hat{v}_{t}\right) d t+2 \sigma \sqrt{\hat{v}_{t}} d \widetilde{B}_{t}^{\hat{v}} .
$$

Comparing (3.2) and (3.3), we conclude that

$$
\hat{\kappa}^{*}=2 \kappa^{*}, \quad \hat{\sigma}=2 \sigma, \quad \hat{\theta}^{*}=\frac{\sigma^{2}}{2 \kappa^{*}} .
$$

To ensure consistency of the two models, another condition $\theta^{*}=0$ must be satisfied.

Assuming $v_{0}=0.2, \kappa^{*}=11.35 / 2, \sigma=0.618 / 2$ and other parameters in the $\mathrm{O}-\mathrm{U}$ process are unchanged, at this moment the associating parameters in the Heston model become $\hat{\kappa}^{*}=11.35, \hat{\sigma}=0.618, \hat{v_{0}}=0.04, \hat{\theta}^{*}=0.0084$, and other parameters in this model are equal to that with the $\mathrm{O}-\mathrm{U}$ process. We substitute these predetermined 


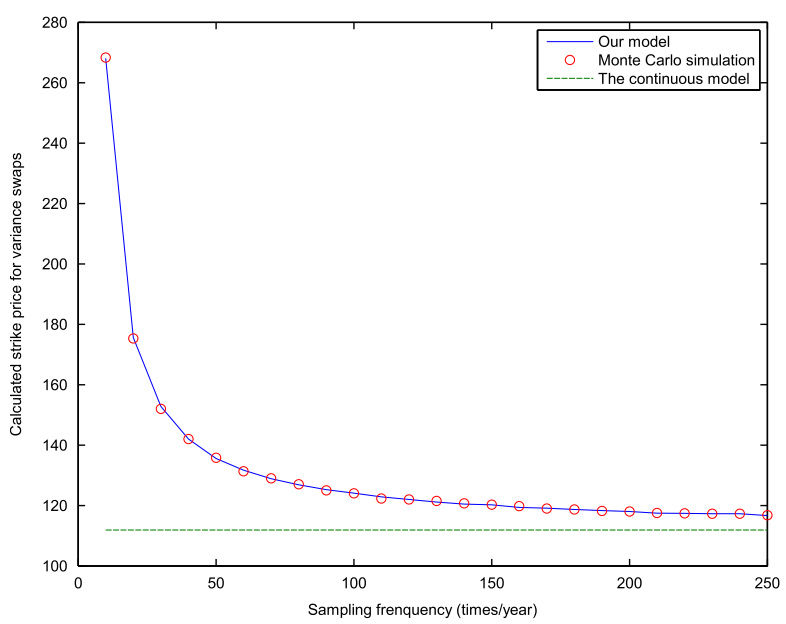

FIGURE 2. A comparison of fair strike values based on the discrete model, continuous model and (3.4).

TABLE 2. The numerical results of the parameters-changed model.

\begin{tabular}{lccc}
\hline Sampling frequency & Discrete model & Continuous model & MC simulation \\
\hline Monthly $(N=12)$ & 234.7829 & 111.8411 & 235.5147 \\
Fortnightly $(N=26)$ & 161.1387 & 111.8411 & 160.3153 \\
Weekly $(N=52)$ & 138.6451 & 111.8411 & 136.5840 \\
Daily $(N=252)$ & 116.8177 & 111.8411 & 116.9222 \\
\hline
\end{tabular}

parameters into the corresponding process and take numerical tests on our discrete model. In this way, the MC algorithm should be based on the following EulerMaruyama discretization for the Heston model

$$
\left\{\begin{array}{l}
S_{t}=S_{t-1}+r S_{t-1} \Delta t+\sqrt{\left|v_{t-1}\right|} S_{t-1} \sqrt{\Delta t} W_{t}^{1} \\
v_{t}=v_{t-1}+\kappa^{*}\left(\theta^{*}-v_{t-1}\right) \Delta t+\sigma \sqrt{\left|v_{t-1}\right|} \sqrt{\Delta t}\left(\rho W_{t}^{1}+\sqrt{1-\rho^{2}} W_{t}^{2}\right)
\end{array}\right.
$$

where $W_{t}^{1}$ and $W_{t}^{2}$ are two independent standard normal random variables. The results of our tests are shown in Figure 2 and Table 2.

From the results in the graph and data, it is clear that the two values of our discrete model and the MC simulation tend to be consistent as shown in Figure 1. Note that these two values have a limit of 111.8411 for the continuous model (see [32]).

3.2. Discussion on the special form of (A2) in Appendix A As mentioned in Section 2.3, several papers (see [22, 29]) pointed out the limitation of the form of the solutions of the Riccati equations. For example, Zhang's paper [29] is also about the $\mathrm{O}-\mathrm{U}$ process, and there is a group of Riccati equations similar to ours:

$$
\frac{d E}{d \tau}=2 \sigma^{2} E^{2}+\left(2 \rho \gamma \sigma-2 \kappa^{*}\right) E+\frac{1}{2} \gamma(\gamma-1) .
$$


TABLE 3. The numerical results of our model and Zhang's model.

\begin{tabular}{lccc}
\hline Sampling frequency & Our discrete model & Zhang's model & MC simulation \\
\hline Quarterly $(N=4)$ & 482.9213 & $481.8+2.0722 e^{-5} i$ & 483.9658 \\
Monthly $(N=12)$ & 460.4125 & $458.5+2.0003 e^{-5} i$ & 461.2613 \\
Weekly $(N=52)$ & 451.0328 & $457.4+1.9593 e^{-5} i$ & 452.1572 \\
Daily $(N=252)$ & 450.1174 & $452.1+1.9594 e^{-5} i$ & 450.7324 \\
\hline
\end{tabular}

If $\left(2 \kappa^{*}-2 \rho \gamma \sigma\right)^{2}-4 \sigma^{2} \gamma(\gamma-1)<0$, the analytical form should change. Otherwise, the last strike price of variance swaps will become a complex value rather than a real number. This is determined by the property of the quadratic differential equation with one variable in the real fields. Although there is a similar group of equations in the deduction process of our model, the strike price of our discrete model will not become a complex number, regardless of the change of parameters. We take for example, $v_{0}=0.2, \kappa^{*}=0.0134, \theta^{*}=0.2, \rho=-0.64, \sigma=0.1, r=0.0953$ and show the results of the strike price of variance swaps of Zhang's [29] and our model in Table 3.

One reason why the strike price of our discrete model will not be a complex number regardless of the change of parameters, is that our differential equation is set up in a complex number field, unlike the models of Rujivan and Zhu [22] and Zhang [29]. In this way, no matter how these parameters change, the solution can be simplified to one form. Although this is suitable for differential equations in a real number field, sometimes the solution is just local instead of being global. For example, the solution of (3.5) is

$$
E(\gamma, \tau)=\frac{1}{4 \sigma^{2}}\left[\sqrt{-\hat{c}(\gamma)} \tan \left(\frac{\sqrt{-\hat{c}(\gamma)}}{2} \tau-\phi(\gamma)\right)+\hat{a}(\gamma)\right],
$$

where $\hat{c}(\gamma)=\left(2 \kappa^{*}-2 \rho \gamma \sigma\right)^{2}-4 \sigma^{2} \gamma(\gamma-1), \quad \hat{a}(\gamma)=2 \kappa^{*}-2 \rho \gamma \sigma$, and $\tau$ is the independent variable. From the solution we known that the range of $\tau$ is $\tau \in$ $[0,(\pi-2 \phi(\gamma)) / \sqrt{-\hat{c}(\gamma)}]$ and $\phi(\gamma)=\arctan (-\hat{a}(\gamma) / \sqrt{-\hat{c}(\gamma)})$. Transforming (3.6) into an exponential form yields

$$
E(\gamma, \tau)=\frac{\gamma(\gamma-1)\left(e^{H \tau}-1\right)}{(G+H) e^{H \tau}-G+H},
$$

where $G=2 \kappa^{*}-2 \rho \gamma \sigma$ and $H=\sqrt{G^{2}-4 \sigma^{2} \gamma(\gamma-1)}$. In equation (3.7), the range of $\tau$ is $\tau \in[0, \infty]$, which is different from that in (3.6). Thus, the procedure of transformation is wrong, while if $\left(2 \kappa^{*}-2 \rho \gamma \sigma\right)^{2}-4 \sigma^{2} \gamma(\gamma-1)>0$, the global solution (3.7) can be obtained without ambiguity. When using (3.6) to calculate the last results in (2.20) a complex value will be obtained, but using (3.7) to calculate the last results will yield a real value.

On the other hand, in some formulas of our model including the derivative of $C(\omega, \tau), D(\omega, \tau), E(\omega, \tau)$ such as $(2.12),(2.13)$ and so on, theoretically there should be complex numbers, because

$$
\left.\frac{\partial B}{\partial \omega}\right|_{\omega=0}=\frac{i\left(\sigma^{2}-2 \sigma \kappa \rho\right)}{2 \kappa} \neq 0,
$$


TABLE 4. The time spent in four approaches (in seconds).

\begin{tabular}{lcccc}
\hline $\begin{array}{l}\text { Sampling } \\
\text { frequency }\end{array}$ & Our model & Zhu's model & Zhang's model & MC simulation \\
\hline Quarterly $(N=4)$ & 0.1600 & 0.1210 & 0.0031 & 0.5930 \\
Monthly $(N=12)$ & 0.4910 & 0.3070 & 0.0060 & 1.8010 \\
Weekly $(N=52)$ & 1.9030 & 1.5120 & 0.0170 & 7.6910 \\
Daily $(N=252)$ & 9.4050 & 7.6910 & 0.0210 & 36.2820 \\
\hline
\end{tabular}

TABLE 5. The sensitivity of the strike price of the variance swap (daily sampling).

\begin{tabular}{ccc}
\hline Parameter & Value & Sensitivity $(\%)$ \\
\hline$\kappa^{*}$ & 11.35 & -0.0375 \\
$\theta^{*}$ & 0.022 & 1.073 \\
$\sigma$ & 0.618 & -0.0264 \\
$v_{0}$ & 0.04 & 0.28 \\
\hline
\end{tabular}

but the last strike price is still a real number. Beside our analysis in Section 2.3, it is known that in our last formula the coefficient of $i$ becomes 0 in the process of integrating polynomials. So, similar to the work of Zhu and Lian [32] and Jia et al. [17], the parameters in our model are allowed to change randomly.

3.3. Efficiency and sensitivity One of the aims is to improve our efficiency to price variance swaps with a dynamic underlying asset. In this section, we show the efficiency of our discrete model. In order to make a comparison conveniently, we show the running time of the kinds of procedures for algorithms that include our model and the models of Zhu and Lian [32], Zhang [29] and the MC simulation in MATLAB in Table 4.

It is clear that the efficiency of the three analytical solutions is better than the MC simulation. The two models using dimension reduction are obviously less efficient than Zhang's method, because the derivation process makes our model much more complex, and the last analytical equation (2.5) in the two models is longer than another formula. In this way, the shortcoming of the two applications of the dimension reduction technique is their running time, especially when the sampling frequency is large. However, compared to the MC algorithm, this method saves time as well.

Finally, to demonstrate how sensitive the strike price is to the change of key parameters in the model, some sensitivity tests are taken for the example presented in this section. The results of the percentage change of the strike price are shown in Table 5, which is caused by model parameters changed by $1 \%$ from their base values used in the example presented in this section. As certain papers showed before, it is clear that the strike price is most sensible to the long-term mean volatility $\theta$ for the cases studied. The spot volatility $v_{0}$ also has an important influence on the strike price, and the least sensible parameter is the mean-reverting speed parameter $\kappa^{*}$. 


\section{Conclusion}

In this paper, we extend the approach proposed by Little and Pant [19] and Zhu and Lian [32] to price variance swaps with the realized variance defined as the average of the squared log returns of the underlying asset, and obtain a closed-form exact solution based on the $\mathrm{O}-\mathrm{U}$ process. This model supports the fact that the method of Zhu and Lian [32] is indeed quite versatile, and can be used for other definitions of the realized variance as well. Based on the relationship between the Heston model and mean-reverting volatility model, we use the MC simulation based on the two dynamic processes to test our model, and find that our model is correct. There is the same procedure which solves our model, and other models such as Zhang [29] and Rujivan and Zhu [22], but our model can avoid the limitation as pointed out in these two articles, and allows parameters to change randomly. Thus, we do not need to worry that the strike price is a local solution in our model. The disadvantage of our model is its long running time; however, it is at least faster than the MC simulation.

There are different stochastic processes consisting of the simultaneous movement of an underlying asset and its volatility. We can extend the applications in pricing variance swaps based on the Heston model into other processes such as Merton's jump-diffusion model [20], Bates and Scott's model [2, 24], and so on. In addition, we may add the movement of stochastic interest rate, and make these kinds of stochastic processes include three variables.

\section{Acknowledgement}

This work was supported by the Major Innovation Projects for Building First-class Universities in China's Western Region (grant no. ZKZD2017009).

\section{Appendix A}

We rewrite the system in Proposition 2.1:

$$
\left\{\begin{array}{l}
\frac{\partial U}{\partial t}+\frac{1}{2} v^{2} S^{2} \frac{\partial^{2} U}{\partial S^{2}}+\rho \sigma v S \frac{\partial^{2} U}{\partial S \partial v}+\frac{1}{2} \sigma^{2} \frac{\partial^{2} U}{\partial v^{2}}+r S \frac{\partial U}{\partial S}+\left[\kappa^{*}\left(\theta^{*}-v\right)\right] \frac{\partial U}{\partial v}-r U=0 \\
U(S, v, T)=H(S)
\end{array}\right.
$$

Firstly, let $\tau=T-t, x=\ln S$, then the converted PDE system can be obtained

$$
\left\{\begin{array}{l}
\frac{\partial U}{\partial \tau}=\frac{1}{2} v^{2} \frac{\partial^{2} U}{\partial x^{2}}+\rho \sigma v \frac{\partial^{2} U}{\partial x \partial v}+\frac{1}{2} \sigma^{2} \frac{\partial^{2} U}{\partial v^{2}}+\left(r-\frac{1}{2} v^{2}\right) \frac{\partial U}{\partial x}+\left[\kappa^{*}\left(\theta^{*}-v\right)\right] \frac{\partial U}{\partial v}-r U \\
U(x, v, 0)=H\left(e^{x}\right) .
\end{array}\right.
$$

Then, we apply the generalized Fourier transform to the PDE with respect to $x$. Before obtaining the solution, the formula for the characteristic of the transform should be introduced. 
THEOREM A.1. If $n>0$ and $n$ is an integer, and $\lim _{|x| \rightarrow \infty} f^{(r)}(x)=0$ for $r=0,1, \ldots, n-1$ with $f^{(0)}(x) \equiv f(x)$, then

$$
\mathcal{F}\left[f^{(n)}(x) ; \xi\right]=(-i \xi)^{n} F(\xi) .
$$

After transform, the corresponding PDE system can be obtained:

$$
\left\{\begin{array}{l}
\frac{\partial \widetilde{U}}{\partial \tau}=\frac{1}{2} \sigma^{2} \frac{\partial^{2} \widetilde{U}}{\partial v^{2}}+\left[-\frac{1}{2} v^{2}\left(\omega^{2}+\omega i\right)+r \omega i-r\right] \widetilde{U} \\
\quad+\left[v\left(\rho \sigma \omega i-\kappa^{*}\right)+\kappa^{*} \theta^{*}\right] \frac{\partial \widetilde{U}}{\partial v} \\
\widetilde{U}(\omega, v, 0)=\mathcal{F}\left[H\left(e^{x}\right)\right] .
\end{array}\right.
$$

Following Rainer Schöbel's [26] deduction process in the mean-reverting stochastic model, we might as well assume

$$
\widetilde{U}(\omega, v, \tau)=e^{C(\omega, \tau)+D(\omega, \tau) v+E(\omega, \tau) v^{2}} \widetilde{U}(\omega, v, 0) .
$$

Let $c_{1}=\left(\omega^{2}+i \omega\right) / 2, \quad b=(i \omega-1) r, A=\kappa-i \rho \sigma \omega, B=\sqrt{A^{2}+2 c_{1} \sigma^{2}}$. Then we substitute this function into (A1) to obtain a Riccati differential equation group:

$$
\left\{\begin{array}{l}
\frac{d E}{d \tau}=2 \sigma^{2} E^{2}-2 A E-c_{1}, \\
\frac{d D}{d \tau}=2 \sigma^{2} D E-A D+2 E \kappa^{*} \theta^{*}, \\
\frac{d C}{d \tau}=\frac{1}{2} \sigma^{2}\left(2 E+D^{2}\right)+\kappa^{*} \theta^{*} D+b
\end{array}\right.
$$

with initial conditions $E(\omega, 0)=0, D(\omega, 0)=0$ and $C(\omega, 0)=0$. The analytical solutions of these equations are given in (2.11). After obtaining the solutions, an inverse Fourier transform should be utilized to get the solution of the original PDE as

$$
\begin{aligned}
U(x, v, \tau) & =\mathcal{F}^{-1}[\widetilde{U}(\omega, v, \tau)] \\
& =\mathcal{F}^{-1}\left[e^{C(\omega, T-t)+D(\omega, T-t) v+E(\omega, T-t) v^{2}} \mathcal{F}\left[H\left(e^{x}\right)\right]\right] .
\end{aligned}
$$

\section{Appendix B}

The Feynman-Kac formula tells us that the solution of PDE (2.14) can be written as a conditional expectation

$$
U(S, v, t)=\mathbb{E}_{t}^{Q}\left[e^{r(T-t)} G\left(v_{T}\right)\right] .
$$

The expectation is actually not related to the process $S_{t}$, since the payoff function is independent of $S$. Thus, we only need to know the distribution of $v_{t}$ to find its expectation. Given that $v_{t}$ follows the dynamic (2.1), we apply the Itô formula to $e^{\kappa^{*} t} v_{t}$

$$
\begin{aligned}
d e^{\kappa^{*} t} v_{t} & =\kappa^{*} e^{\kappa^{*} t} v_{t} d t+e^{\kappa^{*} t}\left[\kappa^{*}\left(\theta^{*}-v_{t}\right) d t+\sigma d \widetilde{B}_{t}^{v}\right] \\
& =\kappa^{*} \theta^{*} e^{\kappa^{*} t} d t+\sigma e^{\kappa^{*} t} d \widetilde{B}_{t}^{v} .
\end{aligned}
$$


Integrating both sides of (B1),

$$
v_{t}=e^{-\kappa^{*} t} v_{0}+\theta^{*}\left(1-e^{-\kappa^{*} t}\right)+\sigma e^{-\kappa^{*} t} \int_{0}^{t} e^{\kappa^{*} s} d \widetilde{B}_{s}^{v} .
$$

Note that the random variable $\int_{0}^{t} e^{k^{*} s} d \widetilde{B}_{s}^{v}$ appearing on the right-hand side of (B2), is normally distributed with a mean of 0 and variance

$$
\int_{0}^{t} e^{2 \kappa^{*} s} d s=\frac{1}{2 \kappa^{*}}\left(e^{2 \kappa^{*} t}-1\right)
$$

Therefore, $v_{t}$ is normally distributed with a mean $e^{-k^{*} t} v_{0}+\theta^{*}\left(1-e^{-k^{*} t}\right)$ and variance $\left(\sigma^{2} /\left(2 \kappa^{*}\right)\right)\left(1-e^{-2 \kappa^{*} t}\right)$.

\section{References}

[1] G. Bakshi, C. Cao and Z. Chen, "Empirical performance of alternative option pricing models", J. Finance 52 (1997) 2003-2049; doi:10.1111/j.1540-6261.1997.tb02749.x.

[2] D. S. Bates, "Jumps and stochastic volatility: exchange rate processes implicit in Deutsche mark options", Rev. Finac. Stud. 9 (1996) 69-107; doi:10.1093/rfs/9.1.69.

[3] F. Black and M. Scholes, "The pricing of options and corporate liabilities", J. Polit. Econ. 81 (1973) 637-654; doi:10.1086/260062.

[4] M. Broadie and A. Jain, "The effect of jumps and discrete sampling on volatility and variance swaps", Int. J. Theor. Appl. Finance 11 (2008) 761-797; doi:10.1142/S0219024908005032.

[5] O. Brockhaus and D. Long, "Volatility swaps made simple", Risk 19 (2000) 92-95, https://www.researchgate.net/publication/248350031_Volatility_Swaps_Made_Simple_Risk.

[6] P. Carr and A. Corso, "Commodity covariance contracting", Energy Risk (2001) 42-45, http://engineering.nyu.edu/files/twrdsfig.pdf.

[7] P. Carr and R. Lee, "Robust replication of volatility derivatives", in: Mathematics in finance working paper series (Courant Institute of Mathematical Sciences, New York University, NY, 2008) $1-48$; https://pdfs.semanticscholar.org/2ea8/6c38b34913eedd05a79c880198a27b824217.pdf.

[8] P. Carr and R. Lee, "Realised volatility and variance: options via swaps", Risk 5 (2007) 76-83, http://citeseerx.ist.psu.edu/viewdoc/download?doi=10.1.1.463.7161\&rep=rep1\&type=pdf.

[9] P. Carr and D. Madan, "Towards a theory of volatility trading", in: Volatility: New estimation techniques for pricing derivatives (Risk Publications, London, 1998) 417-427; doi:10.1017/CBO9780511569708.013.

[10] K. Demeterfi, E. Derman, M. Kamal and J. Zou, "More than you ever wanted to know about volatility swaps", Technical Report, Goldman Sachs Quantitative Strategies Research Notes, 1999, 1-52, http://www.emanuelderman.com/writing/entry/more-than-you-ever-wantedto-know-about-volatility-swaps-the-journal-of-der.

[11] R. Elliott, T. Siu and L. Chan, "Pricing volatility swaps under Heston's stochastic volatility model with regime switching", Appl. Math. Finance 14(1) (2007) 41-62; doi:10.1080/13504860600659222.

[12] M. B. Garman, "A general theory of asset valuation under diffusion state processes", in: Research program in finance working paper (Center for Research in Management Science, Berkley, CA, 1976), http://EconPapers.repec.org/RePEc:ucb:calbrf:50.

[13] A. Grunbichler and F. Longstaff, "Valuing futures and options on volatility", J. Banking Finance 20 (1996) 985-1001; doi:10.1016/0378-4266(95)00034-8.

[14] S. L. Heston, "A closed-form solution for options with stochastic volatility with applications to bond and currency options", Rev. Financ. Stud. 6 (1993) 327-343; doi:10.1093/rfs/6.2.327. 
[15] S. L. Heston and S. Nandi, "Derivatives on volatility: some simple solutions based on observables", in: Working paper series (Federal Reserve Bank of Atlanta, GA, 2000) 1-19; https://pdfs.semanticscholar.org/d2db/dfa9b5957efb5f78657c6aded9da1bcbe57b.pdf.

[16] A. Javaheri, P. Wilmott and E. Haug, "GARCH and volatility swaps", Quant. Finance 4 (2004) 589-595; doi:10.1080/14697680400000040.

[17] Z. L. Jia, X. C. Bi and S. G. Zhang, "Pricing variance swaps under stochastic volatility with an Ornstein-Uhlenbeck process", J. Syst. Complex 28 (2015) 1412-1425; doi:10.1007/s11424-015-3165-6.

[18] G. H. Lian, C. Chiarella and P. S. Kalev, "Volatility swaps and volatility options on discretely sampled realized variance", J. Dynamics Control 47 (2014) 239-262; doi:10.1016/j.jedc.2014.08.014.

[19] T. Little and V. Pant, "A finite-difference method for the valuation of variance swaps", J. Comput. Finance 5 (2001) 81-103; doi:10.21314/JCF.2001.057.

[20] R. Merton, “The theory of rational option pricing”, Bell J. Econom. Manage Sci. 1 (1973) 141-183; doi: $10.2307 / 3003143$.

[21] A. D. Poularikas, The transforms and applications handbook, 2nd edn The electrical engineering handbook series (CRC Press LLC, Boca Raton, 2000).

[22] S. Rujivan and S. P. Zhu, "A simplified analytical approach for pricing discretely-sampled variance swaps with stochastic volatility", Appl. Math. Lett. 25 (2012) 1644-1650; doi:10.1016/j.aml.2012.01.029.

[23] S. Rujivan and S. P. Zhu, "A simple closed-form formula for pricing discretely-sampled variance swaps under the Heston model", ANZIAM J. 56 (2014) 1-27; doi:10.21914/anziamj.v56i0.7455.

[24] L. O. Scott, "Pricing stock options in a jump diffusion model with stochastic volatility and interest rates: applications of Fourier inversion methods", Math. Finance 7 (1997) 413-426; doi:10.1111/1467-9965.00039.

[25] A. Sepp, "Pricing options on realized variance in the Heston model with jumps in returns and volatility", J. Comput. Finance 11 (2008) 33-70; doi:10.21314/JCF.2008.185.

[26] E. M. Stein and J. E. Stein, "Stock price distributions with stochastic volatility and interest rates", Rev. Financ. Stud. 4 (1991) 727-752; doi:10.1093/rfs/4.4.727.

[27] A. Swishchuk, "Modeling of variance and volatility swaps for financial markets with stochastic volatilities, Wilmott magazine September issue", Technical Article 2 (2004) 64-72; http://math.ucalgary.ca/files/finlab/StochVolatSwap.pdf.

[28] H. Windcliff, P. Forsyth and K. Vetzal, "Pricing methods and hedging strategies for volatility derivatives", J. Banking Finance 30 (2006) 409-431; doi:10.1016/j.jbankfin.2005.04.025.

[29] L.-W. Zhang, "A closed-form pricing formula for variance swaps with mean-reverting Gaussian volatility", ANZIAM J. 55 (2014) 362-382; doi:10.1017/S144618111400011X.

[30] W. D. Zheng and Y. K. Kwok, "Closed form pricing formulas for discretely sampled generalized variance swaps", Math. Finance 24 (2014) 855-881; doi:10.1111/mafi.12016.

[31] S. P. Zhu and G. H. Lian, "A closed-form exact solution for pricing variance swaps with stochastic volatility", Math. Finance 21 (2011) 233-256; doi:10.1111/j.1467-9965.2010.00436.x.

[32] S. P. Zhu and G. H. Lian, "On the valuation of variance swaps with stochastic volatility", Appl. Math. Comput. 219 (2012) 1654-1669; doi:10.1016/j.amc.2012.08.006.

[33] S. P. Zhu and G. H. Lian, "Pricing forward-start variance swaps with stochastic volatility", Appl. Math. Comput. 250 (2015) 920-933; doi:10.1016/j.amc.2014.10.050. 